\title{
Genus distribution of graph amalgamations: Pasting when one root has arbitrary degree
}

\author{
Imran F. Khan, Mehvish I. Poshni , Jonathan L. Gross \\ Columbia University, Department of Computer Science NY 10027, New York, USA
}

Received 2 July 2009, accepted 14 July 2010, published online 28 August 2010

\begin{abstract}
This paper concerns counting the imbeddings of a graph in a surface. In the first installment of our current work, we showed how to calculate the genus distribution of an iterated amalgamation of copies of a graph whose genus distribution is already known and is further analyzed into a partitioned genus distribution (which is defined for a double-rooted graph). Our methods were restricted there to the case with two 2 -valent roots. In this sequel we substantially extend the method in order to allow one of the two roots to have arbitrarily high valence.
\end{abstract}

Keywords: Graph, genus distribution, vertex-amalgamation.

Math. Subj. Class.: 05C10

\section{Introduction}

We continue the development of methods for counting imbeddings of interesting families of graphs in a range of surfaces. We are primarily concerned here with deriving recursions, rather than with exact formulas. It may be helpful to precede the reading of this paper with a light reading of [13].

By the vertex-amalgamation of the rooted graphs $(G, t)$ and $(H, u)$, we mean the graph obtained from their disjoint union by merging the roots $t$ and $u$. We denote the operation of amalgamation by an asterisk, i.e.,

$$
(G, t) *(H, u)=(X, w)
$$

where $X$ is the amalgamated graph and $w$ the merged root.

Terminology. We take a graph to be connected and an imbedding to be cellular and orientable, unless it is evident from context that something else is intended. A graph need

E-mail addresses: imran@cs.columbia.edu (Imran F. Khan), poshni@cs.columbia.edu (Mehvish I. Poshni), gross@cs.columbia.edu (Jonathan L. Gross) 
not be simple, i.e., it may have self-loops and multiple edges between two vertices. We use the words degree and valence of a vertex to mean the same thing. Each edge has two edge-ends, in the topological sense, even if it has only one endpoint.

Abbreviation. We abbreviate face-boundary walk as $f b$-walk.

Notation. The degree of a vertex $y$ is denoted $\operatorname{deg}(y)$. The genus of a surface $S$ is denoted $\gamma(S)$. The number of imbeddings of a graph $G$ in the surface $S_{i}$ of genus $i$ is denoted $g_{i}$. The sequence $\left\{g_{i}(G) \mid i \geq 0\right\}$ is called the genus distribution of the graph $G$. The terminology generally follows [15] and [1]. For additional background, see [3], [24], or [34].

Prior work concerned with the number of imbeddings of a graph in a minimum-genus surface includes [2], [8], [9], and [19]. Prior work concerned with counting imbeddings in all orientable surfaces or in all surfaces includes [4], [5], [7], [12], [14], [20], [21], [22], [23], [25], [27], [28], [29], [30], [31], [32], and [33].

Remark 1.1. Some of the calculations in this paper are quite intricate, and it appears that taking the direct approach here to amalgamating two graphs at roots of arbitrarily high degree might be formidable. We observe that a vertex of arbitrary degree can be split (by inverse contraction) into two vertices of smaller degree. Effects on the genus distribution that arise from splitting a vertex are described by [11].

\section{Imbeddings induced by an amalgamation of two imbedded graphs}

We say that the pair of imbeddings $\iota_{G}: G \rightarrow S_{G}$ and $\iota_{H}: H \rightarrow S_{H}$ induce the set of imbeddings of $X=G * H$ whose rotations have the same cyclic orderings as in $G$ and $H$, and that this set of imbeddings of $X$ is the result of amalgamating the two imbeddings $\iota_{G}: G \rightarrow S_{G}$ and $\iota_{H}: H \rightarrow S_{H}$.

Proposition 1.2. For any two imbeddings $\iota_{G}: G \rightarrow S_{G}$ and $\iota_{H}: H \rightarrow S_{H}$ of graphs into surfaces, the cardinality of the set of imbeddings of the amalgamated graph $(X, w)=$ $(G, t) *(H, u)$ whose rotation systems are consistent with the imbeddings $\iota_{G}: G \rightarrow S_{G}$ and $\iota_{H}: H \rightarrow S_{H}$ is

$$
(\operatorname{deg}(u)+\operatorname{deg}(t)-1) \cdot\left(\begin{array}{c}
\operatorname{deg}(t)+\operatorname{deg}(u)-2 \\
\operatorname{deg}(u)-1
\end{array}\right)
$$

Proof. Formula (1.1) is a symmetrization of Formula (1.1) of [13].

In the amalgamation $(G, t) *(H, u)=(X, w)$, when one of the roots $t$ and $u$ is 1 -valent, the genus distribution of the resulting graph is easily derivable via bar-amalgamations (see [12]). For the case where

$$
\operatorname{deg}(t)=\operatorname{deg}(u)=2,
$$

methods for calculating the genus distribution are developed in [13]. For the purposes of this paper, we assume that $\operatorname{deg}(t)=2$ and $\operatorname{deg}(u)=n \geq 2$. A pair of such imbeddings $\iota_{G}: G \rightarrow S_{G}$ and $\iota_{H}: H \rightarrow S_{H}$ induce, in accordance with Formula (1.1), $n^{2}+n$ imbeddings of the amalgamated graph $X$. We observe that for each such imbedding $\iota_{X}$ : $X \rightarrow S_{X}$, we have

$$
\gamma\left(S_{X}\right)=\left\{\begin{array}{l}
\gamma\left(S_{G}\right)+\gamma\left(S_{H}\right) \text { or } \\
\gamma\left(S_{G}\right)+\gamma\left(S_{H}\right)+1
\end{array}\right.
$$


Terminology. The difference $\gamma\left(S_{X}\right)-\left(\gamma\left(S_{G}\right)+\gamma\left(S_{H}\right)\right)$ is called the genus increment of the amalgamation, or more briefly, the genus increment or increment.

Proposition 1.3. In any vertex-amalgamation $(G, t) *(H, u)=(X, w)$ of two graphs, the increment of genus lies within these bounds:

$$
\left\lceil\frac{1-\operatorname{deg}(t)-\operatorname{deg}(u)}{2}\right\rceil \leq \gamma\left(S_{X}\right)-\left(\gamma\left(S_{G}\right)+\gamma\left(S_{H}\right)\right) \leq\left\lfloor\frac{\operatorname{deg}(t)+\operatorname{deg}(u)-2}{2}\right\rfloor
$$

Proof. See [13].

\section{Double-rooted graphs}

By a double-rooted graph $(H, u, v)$ we mean a graph with two vertices designated as roots. Double-rooted graphs were first introduced in [13] as they lend themselves natrually to iterated amalgmation. For the purposes of this paper, root $u$ is assumed to have degree $n \geq$ 2 , whereas root $v$ is 2-valent. Our focus here, is the graph amalgamation $(G, t) *(H, u, v)$ when $\operatorname{deg}(t)=\operatorname{deg}(v)=2$ and $\operatorname{deg}(u)=n \geq 2$. This is illustrated in Figure 1 .
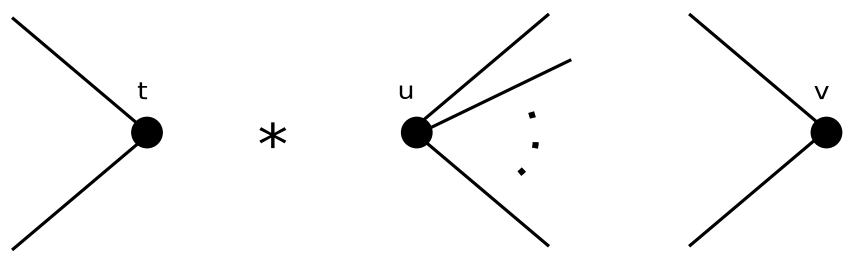

Figure 1: $(G, t) *(H, u, v)$ when $\operatorname{deg}(t)=\operatorname{deg}(v)=2$ and $\operatorname{deg}(u)=n \geq 2$.

When two single-rooted graphs are amalgamated, the amalgamated graph has the merged vertices of amalgamation as its root. If we iteratively amalgamate several single-rooted graphs, we obtain a graph with a root of whose degree is the sum of the degrees of the constituent roots. We use double-rooted graphs when we want to calculate the genus distribution of a chain of copies (as in $\S 3$ and $\S 4$ ) of the same graph (or of different graphs).

\section{Double-root partials and productions}

The genus distribution of the set of imbeddings of $(X, w)=(G, t) *(H, u)$ whose rotation systems are consistent with those of fixed imbeddings $G \rightarrow S_{G}$ and $H \rightarrow S_{H}$, depends only on $\gamma\left(S_{G}\right), \gamma\left(S_{H}\right)$, and the respective numbers of faces of the imbeddings $G \rightarrow S_{G}$ and $H \rightarrow S_{h}$ in which the two vertices of amalgamation $t$ and $u$ lie. Accordingly, we partition the imbeddings of a single-rooted graph $(G, t)$ with $\operatorname{deg}(t)=2$ in a surface of genus $i$ into the subset of type- $d_{i}$ imbeddings, in which root $t$ lies on two distinct fb-walks, and the subset of type- $s_{i}$ imbeddings, in which root $t$ occurs twice on the same fb-walk. Moreover, we define

$$
\begin{aligned}
& d_{i}(G, t)=\text { the number of imbeddings of type- } d_{i}, \text { and } \\
& s_{i}(G, t)=\text { the number of imbeddings of type- } s_{i} .
\end{aligned}
$$


Thus,

$$
g_{i}(G, t)=d_{i}(G, t)+s_{i}(G, t)
$$

The numbers $d_{i}(G, t)$ and $s_{i}(G, t)$ are called single-root partials. The sequences $\left\{d_{i}(G, t) \mid\right.$ $i \geq 0\}$ and $\left\{s_{i}(G, t) \mid i \geq 0\right\}$ are called partial genus distributions.

Since $\operatorname{deg}(u)=n$ in a double-rooted graph $(H, u, v)$, there are $n$ face corners incident at $u$ (i.e., $u$ occurs $n$ times in the fb-walks - we will call them $u$-corners from now on), some or all of which might belong to the same face.

Suppose further that the $n$ occurrences of root $u$ in fb-walks of different faces are distributed according to the partition $p_{1} p_{2} \cdots p_{r}$ of $\mathrm{n}$ (where $r$ is the number of faces incident at root $u$ ). For each such partition $p_{1} p_{2} \cdots p_{r}$, we define the following double-root partials of the genus distribution of a graph $(H, u, v)$, such that root $u$ is $n$-valent and root $v$ is 2-valent:

$$
\begin{aligned}
& f_{p_{1} p_{2} \cdots p_{r}} d_{i}= \begin{array}{l}
\text { the number of imbeddings of } H \text { such that the } n \text { occurrences of } \\
\text { root } u \text { are distributed according to the partition } p_{1} p_{2} \cdots p_{r}, \text { and } \\
\text { the } 2 \text { occurrences of } v \text { lie on two different fb-walks. }
\end{array} \\
& f_{p_{1} p_{2} \cdots p_{r}} s_{i}=\quad \begin{array}{l}
\text { the number of imbeddings of } H \text { such that the } n \text { occurrences of } \\
\text { root } u \text { are distributed according to the partition } p_{1} p_{2} \cdots p_{r}, \text { and } \\
\text { the } 2 \text { occurrences of } v \text { lie on the same fb-walk. }
\end{array}
\end{aligned}
$$

Notation. We write the partition $p_{1} p_{2} \cdots p_{r}$ of an integer in non-ascending order.

A production for an amalgamation

$$
(G, t) *(H, u, v)=(X, v)
$$

of a single-rooted graph $(G, t)$ with a double-rooted graph $(H, u, v)$ (where $\operatorname{deg}(t)=$ $\operatorname{deg}(v)=2$, and $\operatorname{deg}(u) \geq 2$ ) is an expression of the form

$$
\begin{aligned}
p_{i}(G, t) * q_{j}(H, u, v) \longrightarrow & \alpha_{1} d_{i+j}(G * H, v)+\alpha_{2} d_{i+j+1}(G * H, v) \\
& +\alpha_{3} s_{i+j}(G * H, v)+\alpha_{4} s_{i+j+1}(G * H, v)
\end{aligned}
$$

where $p_{i}$ is a single-root partial and $q_{j}$ is a double-root partial, and where $\alpha_{1}, \alpha_{2}, \alpha_{3}$, and $\alpha_{4}$ are integers. It means that amalgamation of a type- $p_{i}$ imbedding of graph $(G, u)$ and a type- $q_{j}$ imbedding of graph $(H, u, v)$ induces a set of $\alpha_{1}$ type- $d_{i+j}, \alpha_{2}$ type- $d_{i+j+1}, \alpha_{3}$ type $-s_{i+j}$, and $\alpha_{4}$ type- $s_{i+j+1}$ imbeddings of $G * H$. We often write such a rule in the form

$$
p_{i} * q_{j} \longrightarrow \alpha_{1} d_{i+j}+\alpha_{2} d_{i+j+1}+\alpha_{3} s_{i+j}+\alpha_{4} s_{i+j+1}
$$

Sub-partials of $f_{p_{1} p_{2} \cdots p_{r}} d_{i}$

In the course of developing productions for amalgamating a single-rooted graph $(G, t)$ to a double-rooted graph $(H, u, v)$, we shall discover that we sometimes need to refine a doubleroot partial into sub-partials. The following two types of numbers are the sub-partials of $f_{p_{1} p_{2} \cdots p_{r}} d_{i}$ : 


$$
\begin{aligned}
& f_{p_{1} p_{2} \cdots p_{r}} d_{i}^{\prime}=\text { the number of type- } f_{p_{1} p_{2} \cdots p_{r}} d_{i} \text { imbeddings such that at } \\
& f_{p_{1} p_{2} \cdots p_{r}} d_{i}^{\left(p_{l}, p_{m}\right)}=\text { the number of type- } f_{p_{1} p_{2} \cdots p_{r}} d_{i} \text { imbeddings such that the }
\end{aligned}
$$

Note that the value of the latter sub-partial of a graph $(H, u, v)$ would be the same for any two pairs $\left(p_{a}, p_{b}\right)$ and $\left(p_{l}, p_{m}\right)$ such that $\left(p_{l}, p_{m}\right)=\left(p_{a}, p_{b}\right)$. Also note that, in general, we have

$$
f_{p_{1} p_{2} \cdots p_{r}} d_{i}=f_{p_{1} p_{2} \cdots p_{r}} d_{i}^{\prime}+\sum_{\substack{\text { over all distinct } \\ \text { pairs }\left(p_{l}, p_{m}\right) \\ \text { with } l<m}} f_{p_{1} p_{2} \cdots p_{r}} d_{i}^{\left(p_{l}, p_{m}\right)}
$$

Example 2.1. For instance, $f_{112} d_{4}=f_{112} d_{4}^{\prime}+f_{112} d_{4}^{(1,1)}+f_{112} d_{4}^{(1,2)}$, since $(1,1)$ and $(1,2)$ are the distinct pairs.

Lemma 2.2. Let $x$ represent a face of an imbedded graph $(H, u, v)$ with $p_{x}>0$ u-corners. There are $p_{x}\left(p_{x}+1\right)$ ways to insert two edge-ends into the u-corners of this face.

Proof. Since there are $p_{x} u$-corners, there are $p_{x}$ choices for the location of the first edgeend. After the first edge-end is inserted, the number of $u$-corners is $p_{x}+1$. Thus, there are $p_{x}+1$ choices for the second edge-end. Hence, there are a total of $p_{x}\left(p_{x}+1\right)$ choices (see Figure 2).

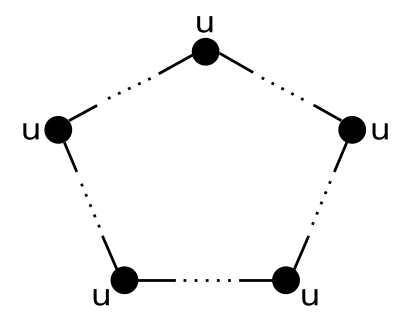

Figure 2: Since $p_{x}=5$, there are $30=5 * 6$ ways to insert two edge-ends into the $u$-corners of this face.

Lemma 2.3. Let $x$ and $y$ be two faces of an imbedded graph $(H, u, v)$, with $p_{x}>0$ and $p_{y}>0 u$-corners, respectively. There are $2 p_{x} p_{y}$ ways to insert two edge-ends at root $u$, such that one edge-end is in face $x$ and the other in face $y$.

Proof. There are $p_{x}$ choices for the edge-end that is inserted into face $x$, and for each such choice, there are $p_{y}$ choices for the other edge-end (see Figure 3). Since either of the two edge-ends can be the one that is inserted into face $x$, we need to multiply $p_{x} p_{y}$ by 2 . 

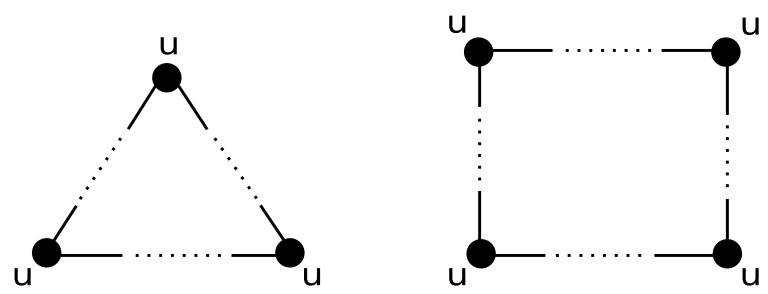

Figure 3: Since $p_{x}=3$ and $p_{y}=4$, there are $24=2 \cdot 3 \cdot 4$ ways to insert two edge-ends with one edge-end in each of the two faces.

Theorem 2.4. Let $p_{1} p_{2} \cdots p_{r}$ be a partition of an integer $n \geq 2$. Suppose that a type- $d_{i}$ imbedding of a single-rooted graph $(G, t)$ is amalgamated to a type- $f_{p_{1} p_{2} \cdots p_{r}} d_{j}$ imbedding of a double-rooted graph $(H, u, v)$, with $\operatorname{deg}(v)=\operatorname{deg}(t)=2$ and $\operatorname{deg}(u)=n$. Then the following production holds:

$$
\begin{aligned}
d_{i} * f_{p_{1} p_{2} \cdots p_{r}} d_{j}^{\prime} & \longrightarrow\left(\sum_{x=1}^{r} p_{x}\left(p_{x}+1\right)\right) d_{i+j} \\
& +\left(\sum_{x=1}^{r} \sum_{y=x+1}^{r} 2 p_{x} p_{y}\right) d_{i+j+1}
\end{aligned}
$$

Proof. Since at most one of the $r$ faces incident at root $u$ of $H$ is incident at root $v$ of $H$, it follows that no matter how the root $t$ of $G$ is amalgamated to $u$, at most one of the two faces incident at $v$ are affected by this amalgamation. It follows that in the amalgamated graph the two occurrences of $v$ remain on two different faces. There are two cases:

case i. Suppose that both edge-ends incident at root $t$ of graph $G$ are placed into one of the $r$ faces of graph $H$ incident at $u$. Then no new handle is needed, and thus, the genus increment is 0 . The coefficient $\sum_{x=1}^{r} p_{x}\left(p_{x}+1\right)$ of $d_{i+j}$ counts the number of ways this can happen. The summation goes from 1 to $r$, since we can put the two edge-ends incident at $t$ into any of the $r$ faces. The term $p_{x}\left(p_{x}+1\right)$ follows from Lemma 2.2.

case ii. Suppose that the two edge-ends incident at root $t$ of graph $G$ are placed into two different faces incident at $u$. This would necessitate adding a handle - resulting in a genus increment of 1 . The coefficient $\sum_{x=1}^{r} \sum_{y=x+1}^{r} 2 p_{x} p_{y}$ of $d_{i+j+1}$ counts the number of ways this can happen, by Lemma 2.3 .

Theorem 2.5. Let $p_{1} p_{2} \cdots p_{r}$ be a partition of an integer $n \geq 2$, and let $\left(p_{l}, p_{m}\right)$ be a pair such that $1 \leq l<m \leq r$. Suppose that a type- $d_{i}$ imbedding of a single-rooted graph $(G, t)$ is amalgamated to a type- $f_{p_{1} p_{2} \cdots p_{r}} d_{j}^{\left(p_{l}, p_{m}\right)}$ imbedding of a double-rooted graph $(H, u, v)$, with $\operatorname{deg}(v)=\operatorname{deg}(t)=2$ and $\operatorname{deg}(u)=n$. Then the following production holds:

$$
\begin{aligned}
d_{i} * f_{p_{1} p_{2} \cdots p_{r}} d_{j}^{\left(p_{l}, p_{m}\right)} & \longrightarrow\left(\sum_{x=1}^{r} p_{x}\left(p_{x}+1\right)\right) d_{i+j} \\
& +\left(\left(\sum_{x=1}^{r} \sum_{y=x+1}^{r} 2 p_{x} p_{y}\right)-2 p_{l} p_{m}\right) d_{i+j+1} \\
& +2 p_{l} p_{m} s_{i+j+1}
\end{aligned}
$$


Proof. Let $\varphi_{l}$ and $\varphi_{m}$ be the two faces incident at root $u$ that are also incident at $v$, with $u$ occurring $p_{l}$ times on fb-walk of face $\varphi_{l}$, and $p_{m}$ times on fb-walk of face $\varphi_{m}$. We note that unless we place one edge-end incident at root $t$ of graph $(G, t)$ into face $\varphi_{l}$ and the other edge-end into face $\varphi_{m}$, at most one of the two faces $\varphi_{l}$ and $\varphi_{m}$ is affected by this amalgamation. Thus, case i remains the same as in Theorem 2.4. The first term of the Production (2.2) reflects this similarity. Moreover, case ii remains the same as in Theorem 2.4, unless $x$ and $y$ correspond to the faces $\varphi_{l}$ and $\varphi_{m}$, which is why we subtract $2 p_{l} p_{m}$ from the second sum in Production (2.2). If $x$ and $y$ correspond to the faces $\varphi_{l}$ and $\varphi_{m}$, then as a result of the amalgamation, the two faces $\left(\varphi_{l}\right.$ and $\left.\varphi_{m}\right)$ combine to become one face having both occurrences of $v$ in its boundary (see Figure 4). The third term of the production reflects this.
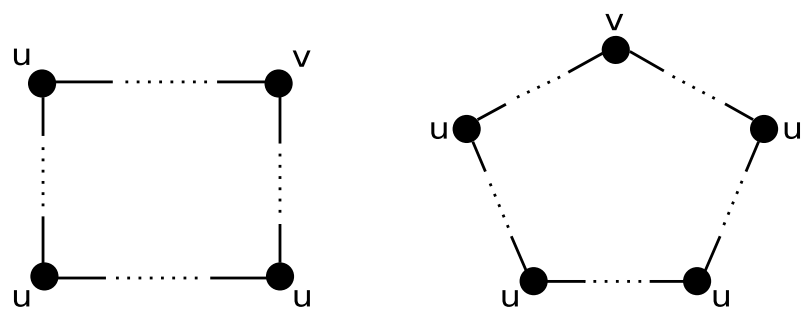

Figure 4: Here $p_{l}=3$ and $p_{m}=4$. Amalgamation combines the two faces, and the resultant face contains both occurrences of $v$.

Notation. We sometimes use the shorthand $f_{p_{1} p_{2} \cdots p_{r}} d_{j}^{\bullet}$ in place of $f_{p_{1} p_{2} \cdots p_{r}} d_{j}$, to emphasize the absence of any superscript after $d_{j}$.

Theorem 2.6. Let $p_{1} p_{2} \cdots p_{r}$ be a partition of an integer $n \geq 2$. Suppose that a type-s $s_{i}$ imbedding of a single-rooted graph $(G, t)$ is amalgamated to a type- $f_{p_{1} p_{2} \cdots p_{r}} d_{j}^{\bullet}$ imbedding of a double-rooted graph $(H, u, v)$, with $\operatorname{deg}(v)=\operatorname{deg}(t)=2$ and $\operatorname{deg}(u)=n$. Then the following production holds:

$$
s_{i} * f_{p_{1} p_{2} \cdots p_{r}} d_{j}^{\bullet} \longrightarrow\left(n^{2}+n\right) d_{i+j}
$$

where the coefficient $n^{2}+n=\left(\begin{array}{c}n+1 \\ n\end{array}\right)$ follows from Proposition 1.2.

Proof. Suppose that in a type- $f_{p_{1} p_{2} \cdots p_{r}} d_{j}^{\bullet}$ imbedding of graph $(H, u, v)$, the two occurrences of root-vertex $v$ lie on on two different fb-walks $W_{1}$ and $W_{2}$ that may or may not contain the root-vertex $u$. Suppose further that the two occurrences of root-vertex $t$ of graph $(G, t)$ lie on fb-walk $X$. The two occurrences of root-vertex $v$ continue being on two different fb-walks after the operation of vertex amalgamation, unless the fb-walks $W_{1}$ and $W_{2}$ combine with the fb-walk $X$ under amalgamation into a single fb-walk. But this cannot happen when the imbedding of $(G, t)$ is a type- $s_{i}$ imbedding, since a reduction of two faces forces the Euler characteristic to be of odd parity, which is not possible. Thus, there is no genus-increment and all $n^{2}+n$ resulting imbeddings are type- $d_{i+j}$ imbeddings. 


\section{Sub-partials of $f_{p_{1} p_{2} \cdots p_{r}} s_{i}$}

To define the sub-partials of $f_{p_{1} p_{2} \cdots p_{r}} s_{i}$ we need the concept of strands, which was introduced and used extensively in [13]. When two imbeddings are amalgamated, these strands recombine with other strands to form new fb-walks.

Definition 2.7. We define a $u$-strand of an fb-walk of a rooted graph $(H, u, v)$ to be a subwalk that starts and ends with the root vertex $u$, such that $u$ does not appear in the interior of the subwalk.

The following two types of numbers are the relevant sub-partials of the partial $f_{p_{1} p_{2} \cdots p_{r}} s_{i}$ for graph $(H, u, v)$ :

$$
\begin{aligned}
& f_{p_{1} p_{2} \cdots p_{r}} s_{i}^{\prime} \quad=\quad \text { the number of type- } f_{p_{1} p_{2} \cdots p_{r}} s_{i} \text { imbeddings of } H \text { such } \\
& \text { that the two occurrences of } v \text { lie in at most one } u \text {-strand. } \\
& f_{p_{1} p_{2} \cdots p_{r}} s_{i}^{\left(p_{l}, c\right)}=\text { the number of type- } f_{p_{1} p_{2} \cdots p_{r}} s_{i} \text { imbeddings of } H \text { such } \\
& \text { that the two occurrences of } v \text { lie in two different } u \text { - } \\
& \text { strands of the fb-walk that is represented by } p_{l} \text {, and such } \\
& \text { that there are } q \geq 1 \text { intermediate } u \text {-corners between } \\
& \text { the two occurrences of } v \text {. We take } c \text { to be equal to } \\
& \min \left(q, p_{l}-q\right) \text {, i.e., equal to the smaller number of inter- } \\
& \text { mediate } u \text {-corners between the two occurrences of root- } \\
& \text { vertex } v \text {. }
\end{aligned}
$$

Note that the last sub-partial would be the same for any other pair $\left(p_{a}, c\right)$ such that $p_{a}=p_{l}$.

Theorem 2.8. Let $p_{1} p_{2} \cdots p_{r}$ be a partition of an integer $n \geq 2$. Suppose that a type- $d_{i}$ imbedding of a single-rooted graph $(G, t)$ is amalgamated to a type- $f_{p_{1} p_{2} \cdots p_{r}} s_{j}^{\prime}$ imbedding of a double-rooted graph $(H, u, v)$, with $\operatorname{deg}(v)=\operatorname{deg}(t)=2$ and $\operatorname{deg}(u)=n$. Then the following production holds:

$$
\begin{aligned}
d_{i} * f_{p_{1} p_{2} \cdots p_{r}} s_{j}^{\prime} & \longrightarrow\left(\sum_{x=1}^{r} p_{x}\left(p_{x}+1\right)\right) s_{i+j} \\
& +\left(\sum_{x=1}^{r} \sum_{y=x+1}^{r} 2 p_{x} p_{y}\right) s_{i+j+1}
\end{aligned}
$$

Proof. Since both occurrences of root $v$ of $H$ lie in at most one $u$-strand of one of the $r$ fb-walks, it follows that regardless of how the $u$-strands recombine in the amalgamation process, these two occurrences remain on that same $u$-strand; thus, in all of the resultant imbeddings, the two occurrences of $v$ are on the same fb-walk. As discussed in the proof of Theorem 2.4, there are $\sum_{x=1}^{r} p_{x}\left(p_{x}+1\right)$ imbeddings that do not result in any genusincrement (corresponding to both edge-ends at $t$ being inserted into the same face at $u$ ), whereas there are $\sum_{y=x+1}^{r} 2 p_{x} p_{y}$ imbeddings that result in a genus increment of 1 (corresponding to inserting both edge-ends at $t$ into the different faces at $u$ ).

Theorem 2.9. Let $p_{1} p_{2} \cdots p_{r}$ be a partition of an integer $n \geq 2$. Then for each distinct $p_{l}$, with $l \in\{1, \cdots, r\}$, and for each integer $c$ in the integer interval $\left[1,\left\lfloor\frac{p_{l}}{2}\right\rfloor\right]$, when $a$ 
type- $d_{i}$ imbedding of a single-rooted graph $(G, t)$ is amalgamated to a type- $f_{p_{1} p_{2} \cdots p_{r}} s_{j}^{\left(p_{l}, c\right)}$ imbedding of a double-rooted graph $(H, u, v)$, with $\operatorname{deg}(v)=\operatorname{deg}(t)=2$ and deg $(u)=n$, the following production holds:

$$
\begin{aligned}
d_{i} * f_{p_{1} p_{2} \cdots p_{r}} s_{j}^{\left(p_{l}, c\right)} & \longrightarrow\left(\left(\sum_{x=1}^{r} p_{x}\left(p_{x}+1\right)\right)-p_{l}\left(p_{l}+1\right)\right) s_{i+j} \\
& +2 c\left(p_{l}-c\right) d_{i+j} \\
& +\left[c(c+1)+\left(p_{l}-c\right)\left(p_{l}-c+1\right)\right] s_{i+j} \\
& +\left(\sum_{x=1}^{r} \sum_{y=x+1}^{r} 2 p_{x} p_{y}\right) s_{i+j+1}
\end{aligned}
$$

Proof. Let $\varphi_{l}$ be the face corresponding to $p_{l}$, and let $w_{1}$ and $w_{2}$ be the two (different) $u$-strands that contain the two occurrences of root $v$ of $H$ (with $c$ intermediate $u$-corners between the two occurrences of $v$ ). It follows that unless the two edge-ends incident at root $t$ of $G$ are both placed into the face $\varphi_{l}$, the two occurrences of root $v$ will lie on the same $\mathrm{fb}$-walk after amalgamation. The first and last terms of the production reflect this.

Now we consider the case when the two edge-ends incident at root $t$ of graph $(G, t)$ are both placed into the face $\varphi_{l}$. Let $e_{\text {start }}$ and $e_{\text {start }}$ be the initial edge-ends of $u$-strands $w_{1}$ and $w_{2}$, similarly let $e_{e n d_{1}}$ and $e_{e n d_{2}}$ be the terminal edge-ends of $u$-strands $w_{1}$ and $w_{2}$ (we consider that a $u$-strand starts and ends at root $u$ ). This is illustrated in Figure 5.

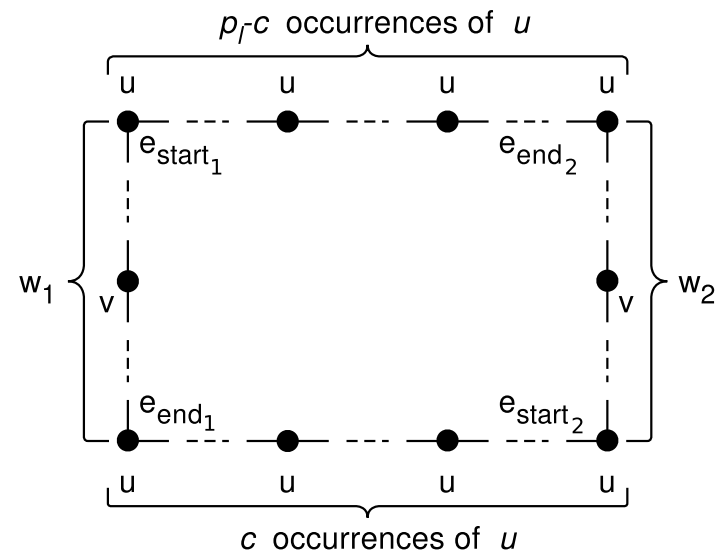

Figure 5: fb-walk of a type- $f_{p_{1} p_{2} \cdots p_{r}} s_{j}^{\left(p_{l}, c\right)}$ imbedding.

It is clear that in the fb-walk of the face $\varphi_{l}$, these four edge-ends appear in $e_{\text {start }}, e_{\text {end }}$, $e_{\text {start }_{2}}, e_{\text {end }_{2}}$ cyclic order. If one of the two edge-ends incident at root $t$ is placed between $e_{\text {end }}$ and $e_{\text {start }}$ and the other between $e_{\text {end }}$ and $e_{\text {start }}$, then after the strands are recombined, one of the $u$-strands containing one occurrence of root $v$ clearly recombines with the one $t$-strand of $(G, t)$ to make a new face (see Figure 6, left).

It follows that in this case the two occurrences of root $v$ will lie on two different faces. Since there are a total of $p_{l} u$-corners in face $\varphi_{l}$, and there are $c$ intermediate $u$-corners between the two occurrences of root $v$ of graph $(H, u, v)$, there are $2 c\left(p_{l}-c\right)$ ways in all 

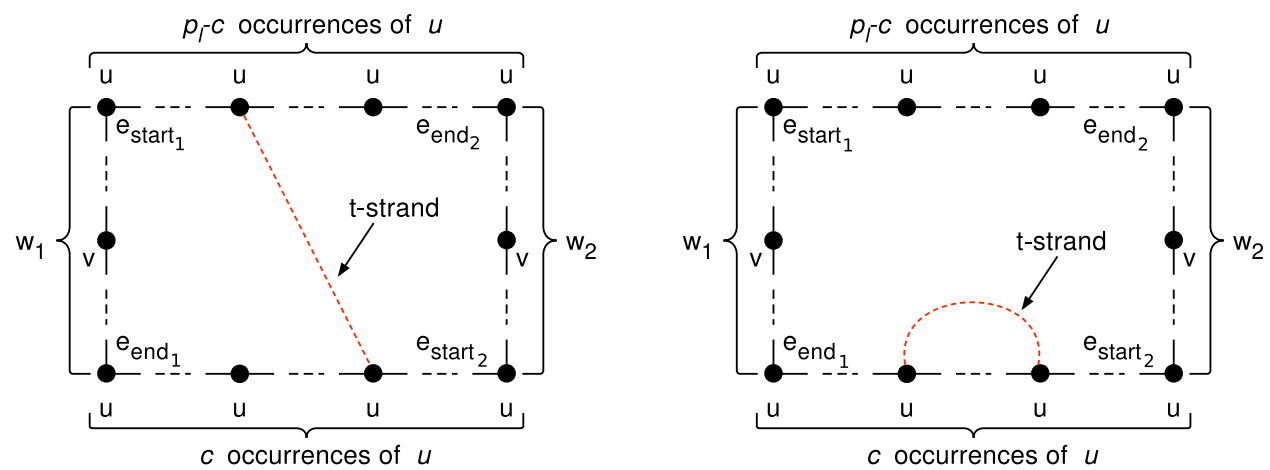

Figure 6: The two ways of inserting $t$-strands.

of inserting the two edge-ends incident at root $t$ of graph $(G, t)$ in this way. We multiply by 2 since either of the two edge-ends can be chosen as the first edge-end. The second term of the production reflects this case.

If both of the edge-ends incident at root $t$ are placed between $e_{e n d_{1}}$ and $e_{\text {start }_{2}}$, or between $e_{e n d_{2}}$ and $e_{\text {start }}$, then the two occurrences of root $v$ lie on the same face after $u$-strands and $t$-strands are recombined (see Figure 6, right). There are $c(c+1)+\left(p_{l}-\right.$ $c)\left(p_{l}-c+1\right)$ ways this can happen, since there are $c$ and $p_{l}-c$ intermediate $u$-corners between $w_{1}$ and $w_{2}$.

Notation. We sometimes use the shorthand $f_{p_{1} p_{2} \cdots p_{r}} s_{j}^{\bullet}$ in place of $f_{p_{1} p_{2} \cdots p_{r}} s_{j}$, to emphasize the absence of any superscript after $s_{j}$.

Theorem 2.10. Let $p_{1} p_{2} \cdots p_{r}$ be a partition of an integer $n \geq 2$. Suppose that a type- $s_{i}$ imbedding of a single-rooted graph $(G, t)$ is amalgamated to a type- $f_{p_{1} p_{2} \cdots p_{r}} s_{j}$ imbedding of a double-rooted graph $(H, u, v)$, with $\operatorname{deg}(v)=\operatorname{deg}(t)=2$ and $\operatorname{deg}(u)=n$. Then the following production holds:

$$
s_{i} * f_{p_{1} p_{2} \cdots p_{r}} s_{j}^{\bullet} \longrightarrow\left(n^{2}+n\right) s_{i+j}
$$

Proof. Since the two occurrences of root $v$ of $H$ lie on the same fb-walk. One necessary condition for the operation of vertex amalgamation to change this is that both edge-ends at root $t$ of $G$ are inserted into that face. However, since both occurrences of root $t$ are on the same fb-walk, both ends of each $t$-strand lie in the same $u$-corner of that face, as illustrated in Figure 7. This implies that no new handle is needed as a result of the amalgamation. Thus, there is no genus-increment.

Corollary 2.11. Let $(X, v)=(G, t) *(H, u, v)$, where $\operatorname{deg}(v)=\operatorname{deg}(t)=2$ and $\operatorname{deg}(u)=$ $n$ for $n \geq 2$. Then for

$$
\alpha_{p_{1} p_{2} \cdots p_{r}}=\sum_{x=1}^{r} p_{x}\left(p_{x}+1\right) \quad \text { and } \quad \beta_{p_{1} p_{2} \cdots p_{r}}=\sum_{x=1}^{r} \sum_{y=x+1}^{r} 2 p_{x} p_{y}
$$

we have 


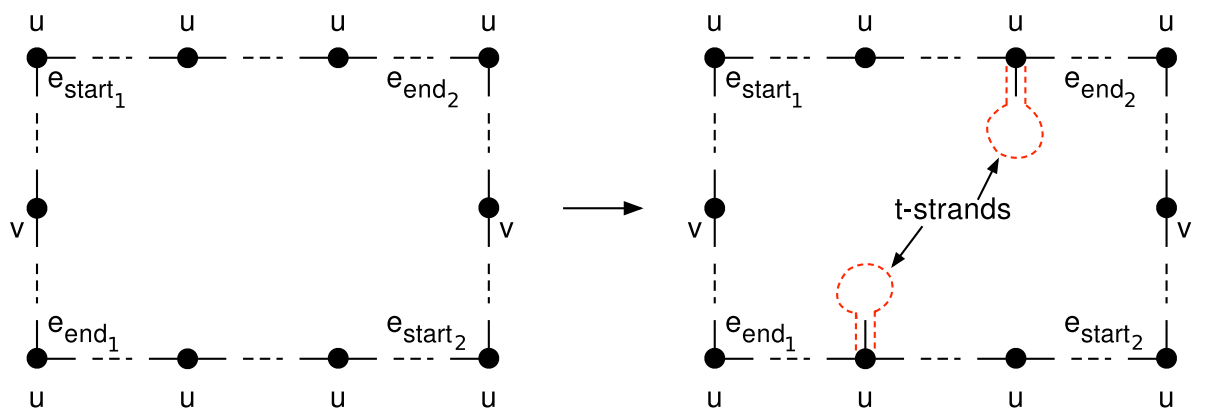

Figure 7: Even after the amalgamation, the two occurrences of $v$ remain on the same fbwalk.

$$
\begin{aligned}
d_{k}(X)= & \sum_{\begin{array}{c}
\text { over all partitions } \\
p_{1} p_{2} \cdots p_{r} \text { of } n
\end{array}}\left[\sum_{i=0}^{k} \alpha_{p_{1} p_{2} \cdots p_{r}} d_{k-i} f_{p_{1} p_{2} \cdots p_{r}} d_{i}^{\prime}\right. \\
& +\sum_{i=0}^{k-1} \beta_{p_{1} p_{2} \cdots p_{r}} d_{k-i-1} f_{p_{1} p_{2} \cdots p_{r}} d_{i}^{\prime} \\
& +\sum_{i=0}^{k} \sum_{\begin{array}{c}
\text { over all } \\
\text { distinct }\left(p_{l}, p_{m}\right)
\end{array}} \alpha_{p_{1} p_{2} \cdots p_{r}} d_{k-i} f_{p_{1} p_{2} \cdots p_{r}} d_{i}^{\left(p_{l}, p_{m}\right)} \\
& +\sum_{i=0}^{k-1} \sum_{\begin{array}{c}
\text { over all } \\
\text { distinct }\left(p_{l}, p_{m}\right) \\
l<m
\end{array}}\left(\beta_{p_{1} p_{2} \cdots p_{r}}-2 p_{l} p_{m}\right) d_{k-i-1} f_{p_{1} p_{2} \cdots p_{r}} d_{i}^{\left(p_{l}, p_{m}\right)} \\
& +\sum_{i=0}^{k}\left(n^{2}+n\right) s_{k-i} f_{p_{1} p_{2} \cdots p_{r}} d_{i}^{\bullet} \\
& \left.+\sum_{i=0}^{k} \sum_{\begin{array}{c}
\text { over all } \\
\text { distinct } p_{l}
\end{array}} \sum_{c=1}^{\left\lfloor\frac{p_{l}}{2}\right\rfloor} 2 c\left(p_{l}-c\right) d_{k-i} f_{p_{1} p_{2} \cdots p_{r}} s_{i}^{\left(p_{l}, c\right)}\right]
\end{aligned}
$$

Proof. This equation is derived from Theorems 2.4, 2.5, 2.6 and 2.9 by a routine transposition of the productions that have the single-root partial $d$ on their right-hand-side.

Corollary 2.12. Let $(X, v)=(G, t) *(H, u, v)$, where $\operatorname{deg}(v)=\operatorname{deg}(t)=2 \operatorname{and} \operatorname{deg}(u)=$ $n$ for $n \geq 2$. Then for

$$
\alpha_{p_{1} p_{2} \cdots p_{r}}=\sum_{x=1}^{r} p_{x}\left(p_{x}+1\right) \quad \text { and } \quad \beta_{p_{1} p_{2} \cdots p_{r}}=\sum_{x=1}^{r} \sum_{y=x+1}^{r} 2 p_{x} p_{y}
$$


we have

$$
\begin{aligned}
& s_{k}(X)=\sum_{\begin{array}{c}
\text { over all partitions } \\
p_{1} p_{2} \cdots p_{r} \text { of } n
\end{array}}\left[\sum_{i=0}^{k} \alpha_{p_{1} p_{2} \cdots p_{r}} d_{k-i} f_{p_{1} p_{2} \cdots p_{r}} s_{i}^{\prime}\right. \\
& +\sum_{i=0}^{k-1} \beta_{p_{1} p_{2} \cdots p_{r}} d_{k-i-1} f_{p_{1} p_{2} \cdots p_{r}} s_{i}^{\prime} \\
& +\sum_{i=0}^{k-1} \sum_{\substack{\text { over all } \\
\text { distinct }\left(p_{l}, p_{m}\right) \\
l<m}} 2 p_{l} p_{m} d_{k-i-1} f_{p_{1} p_{2} \cdots p_{r}} d_{i}^{\left(p_{l}, p_{m}\right)} \\
& +\sum_{i=0}^{k} \sum_{\begin{array}{c}
\text { over all } \\
\text { distinct } p_{l}
\end{array}} \sum_{c=1}^{\left\lfloor\frac{\left.p_{l}\right\rfloor}{2}\right\rfloor}\left(c(c+1)+\left(p_{l}-c\right)\left(p_{l}-c+1\right)\right. \\
& \left.+\alpha_{p_{1} p_{2} \cdots p_{r}}-p_{l}\left(p_{l}+1\right)\right) d_{k-i} f_{p_{1} p_{2} \cdots p_{r}} s_{i}^{\left(p_{l}, c\right)} \\
& +\sum_{i=0}^{k-1} \sum_{\begin{array}{c}
\text { over all } \\
\text { distinct } p_{l}
\end{array}} \sum_{c=1}^{\left\lfloor\frac{p_{l}}{2}\right\rfloor} \beta_{p_{1} p_{2} \cdots p_{r}} d_{k-i-1} f_{p_{1} p_{2} \cdots p_{r}} s_{i}^{\left(p_{l}, c\right)} \\
& \left.+\sum_{i=0}^{k}\left(n^{2}+n\right) s_{k-i} f_{p_{1} p_{2} \cdots p_{r}} s_{i}^{\bullet}\right]
\end{aligned}
$$

Proof. This equation is derived from Theorems 2.5, 2.8, 2.9 and 2.10 by a routine transposition of the productions that have the single-root partial $s$ on their right-hand-side.

Remark 2.13. In writing Recursions 2.7 and 2.8 , we have suppressed indication of graphs $G$ and $H$ as arguments, in order that they not occupy too many lines. In the examples to follow, we see how restriction of these recursions to particular genus distributions of interest greatly simplifies them. The reason for placing the index variable $i$ of each sum with the second factor, rather than the first, also becomes clear in the applications.

\section{Open chains of copies of $K_{4}$}

We can specify a sequence of open chains of copies of a double-rooted graph $(G, u, v)$ recursively.

$$
\begin{aligned}
\left(X_{1}, t_{1}\right) & =(G, v) \quad(\text { suppressing co-root } u) \\
\left(X_{m}, t_{m}\right) & =\left(X_{m-1}, t_{m-1}\right) *(G, u, v) \text { for } m \geq 1
\end{aligned}
$$

For example, consider a chain of copies of the graph $K_{4}$ with one edge subdivided as in Figure 8. We observe that each of the amalgamations results in a vertex of degree 5 .

By face-tracing the imbeddings of $K_{4}$, we obtain Table 1.

By using Recurrences (2.7) and (2.8) for $\operatorname{deg}(u)=n=3$, and the values from Table 1, 

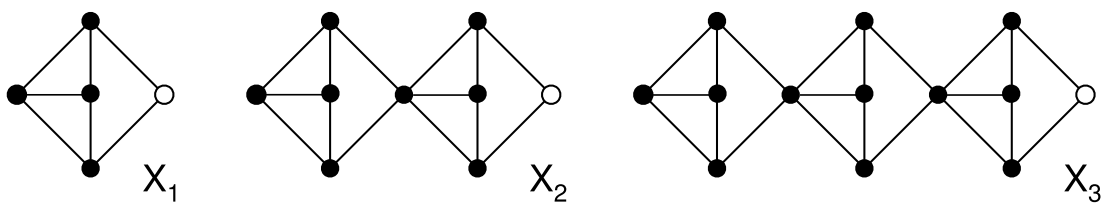

Figure 8: $X_{m}$ is an open chain of $m$ copies of $K_{4}$.

\begin{tabular}{|c|cccc|cc|c|}
\hline \hline$k$ & $f_{111} d^{\prime}{ }_{k}$ & $f_{21} d_{k}^{(2,1)}$ & $f_{21} s_{k}^{(2,1)}$ & $f_{3} d^{\prime}{ }_{k}$ & $d_{k}$ & $s_{k}$ & $g_{k}$ \\
\hline 0 & 2 & 0 & 0 & 0 & 2 & 0 & 2 \\
1 & 0 & 6 & 6 & 2 & 8 & 6 & 14 \\
\hline \hline
\end{tabular}

Table 1: Nonzero partials of $(G, u, v)$.

we obtain the following two recurrences, for $m \geq 2, k \geq 0$ :

$$
\begin{aligned}
& d_{k}\left(X_{m}\right)=12 d_{k}\left(X_{m-1}\right)+24 s_{k}\left(X_{m-1}\right)+96 d_{k-1}\left(X_{m-1}\right)+96 s_{k-1}\left(X_{m-1}\right) \\
& s_{k}\left(X_{m}\right)=48 d_{k-2}\left(X_{m-1}\right)+36 d_{k-1}\left(X_{m-1}\right)+72 s_{k-1}\left(X_{m-1}\right)
\end{aligned}
$$

Another way of obtaining these recurrences without having to use Recurrences (2.7) and (2.8), is to first list all productions that are relevant for the example at hand (i.e. corresponding to the non-zero double-root partials) using Theorems 2.4-2.10; we list the productions for this example in Table 2. We can then transpose these productions, and use the values of double-root partials from Table 1 on the transposed productions to come up with the desired recurrences.

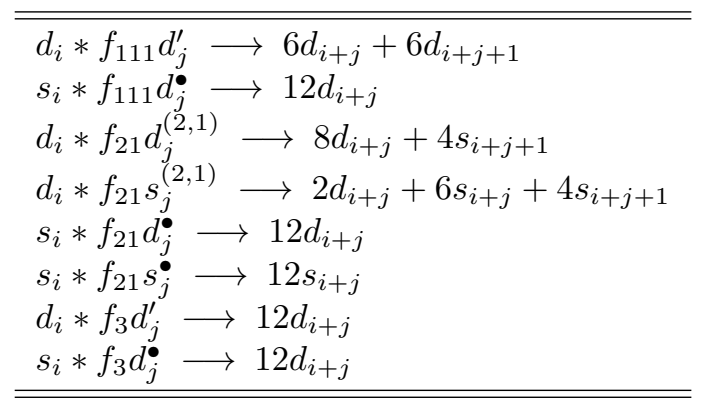

Table 2: The non-zero productions when $\operatorname{deg}(u)=3$.

Using these recurrences and the values of single-root partials in Table 1, we obtain the values of single-root partials for $X_{2}$, that are listed in Table 3 . We can then use values of the partials for $X_{2}$ to obtain the values of single-root partials for $X_{3}$, also listed in Table 3 . We can iterate this to obtain the genus distribution of $X_{m}$ for any value of $m$. 


\begin{tabular}{|r|rr|r||rr|r|}
\hline \hline \multicolumn{5}{|c||}{$X_{2}$} & \multicolumn{3}{c|}{$X_{3}$} \\
\hline$k$ & $d_{k}$ & $s_{k}$ & $g_{k}$ & $d_{k}$ & $s_{k}$ & $g_{k}$ \\
\hline 0 & 24 & 0 & 24 & 288 & 0 & 288 \\
1 & 432 & 72 & 504 & 9216 & 864 & 10080 \\
2 & 1344 & 816 & 2160 & 84096 & 21888 & 105984 \\
3 & 0 & 384 & 384 & 216576 & 127872 & 344448 \\
4 & & & & 36864 & 92160 & 129024 \\
\hline \hline
\end{tabular}

Table 3: Single-root partials of $X_{2}$ and $X_{3}$.

\section{Another example}

As another illustration of the method, we compute the recurrences for the open chains of a graph $(G, s, t)$ in which $\operatorname{deg}(u)=n=6$ (see Figure 9). Where, as in previous example, $X_{1}$ is the graph $G$ with root $s$ suppressed.
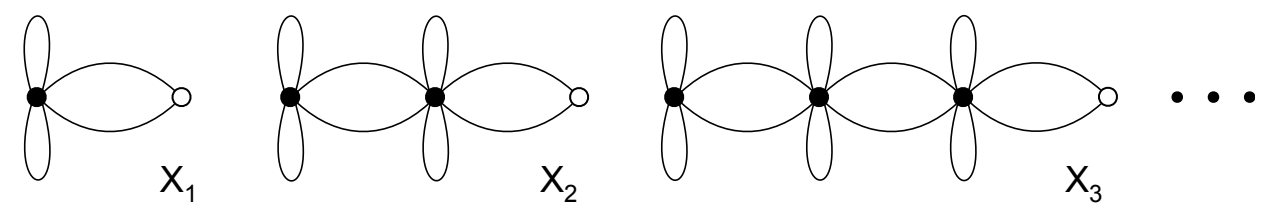

Figure 9: $X_{m}$ is an open chain of $m$ copies of $G$.

By face-tracing the imbeddings of $(G, u, v)$, we obtain Table 4 .

\begin{tabular}{|c|cc|}
\hline \hline type & $k=0$ & $k=1$ \\
\hline$f_{51} d^{(5,1)}$ & 0 & 16 \\
$f_{2211} d^{(2,2)}$ & 8 & 0 \\
$f_{2211} d^{(2,1)}$ & 16 & 0 \\
$f_{42} s^{(4,2)}$ & 0 & 8 \\
$f_{42} d^{(4,2)}$ & 0 & 16 \\
$f_{33} d^{(3,3)}$ & 0 & 8 \\
$f_{3111} d^{(3,1)}$ & 16 & 0 \\
$f_{51} s^{(5,2)}$ & 0 & 32 \\
\hline$d_{k}$ & 40 & 40 \\
$s_{k}$ & 0 & 40 \\
\hline$g_{k}$ & 40 & 80 \\
\hline \hline
\end{tabular}

Table 4: Nonzero partials of $(G, u, v)$.

Using Recurrences (2.7) and (2.8), we obtain the following two recurrences for $m \geq$ 
$2, k \geq 0$ :

$$
\begin{aligned}
d_{k}\left(X_{m}\right)= & 672 d_{k}\left(X_{m-1}\right)+1680 s_{k}\left(X_{m-1}\right) \\
& +2352 d_{k-1}\left(X_{m-1}\right)+1680 s_{k-1}\left(X_{m-1}\right) \\
s_{k}\left(X_{m}\right)= & 1008 d_{k-2}\left(X_{m-1}\right)+1008 d_{k-1}\left(X_{m-1}\right)+1680 s_{k-1}\left(X_{m-1}\right)
\end{aligned}
$$

Table 5 records the values that these recurrences give us for $X_{2}$ and $X_{3}$.

\begin{tabular}{|r|rr|r||rr|r|}
\hline \hline \multicolumn{5}{|c||}{$X_{2}$} & \multicolumn{3}{c|}{$X_{3}$} \\
\hline$k$ & $d_{k}$ & $s_{k}$ & $g_{k}$ & $d_{k}$ & $s_{k}$ & $g_{k}$ \\
\hline 0 & 26880 & 0 & 26880 & 18063360 & 0 & 18063360 \\
1 & 188160 & 40320 & 228480 & 257402880 & 27095040 & 284497920 \\
2 & 161280 & 147840 & 309120 & 867041280 & 284497920 & 1151539200 \\
3 & 0 & 40320 & 40320 & 695439360 & 600606720 & 1296046080 \\
4 & & & & 67737600 & 230307840 & 298045440 \\
\hline \hline
\end{tabular}

Table 5: Single-root partials of $X_{2}$ and $X_{3}$.

\section{Conclusions}

Results in this paper enable us to compute genus distributions of open chains in which the degree of the amalgamated vertex can be arbitrarily large, a significant improvement from the previous results where it was limited to being 4-valent.

Using the methods developed in this paper, one can compute the following:

- the genus distribution of the vertex-amalgamation of two single-rooted graphs $(G, t)$ and $(H, u)$, when $\operatorname{deg}(t)=2$ and $\operatorname{deg}(u)$ is arbitrarily large. One simply adds up all terms on the right-hand-sides of Recurrences (2.7) and (2.8) after converting doubleroot partials to single-root partials (by ignoring the second root).

- recurrences for the genus distribution of the sequence of open chains of doublerooted graphs $(H, u, v)$, where $\operatorname{deg}(v)=2$ and $\operatorname{deg}(u)$ can be arbitrarily large, provided that the genus distribution of $(H, u, v)$ is known and is further analyzed into a partitioned genus distribution.

It is interesting to note that extending the methods developed in this paper to amalgamating a single-rooted graph $(G, t)$ with a double-rooted graph $(H, u, v)$, with $\operatorname{deg}(t) \geq 3$ and $\operatorname{deg}(v) \geq 3$, might not be so straight-forward. As illustrated in Example 5.1, the genusincrement may sometimes be negative (see Research Problem 1 below).

Example 5.1. Figure 10 shows two toroidal imbeddings $\iota_{1}:\left(D_{3}, u\right) \rightarrow S_{1}$ and $\iota_{2}$ : $\left(D_{3}, u\right) \rightarrow S_{1}$ of the single-rooted dipole $\left(D_{3}, u\right)$.

One of the 40 imbeddings of the amalgamated graph $X=\left(D_{3}, u\right) *\left(D_{3}, u\right)$ that is consistent with those two imbeddings is also shown in the figure. Note that this is also a toroidal imbedding, since

$$
V-E+F=3-6+3=0 .
$$




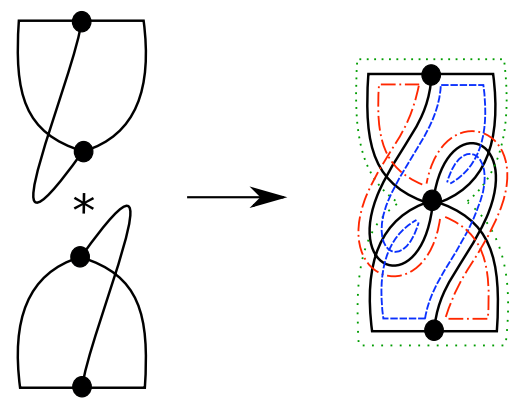

Figure 10: A consistent imbedding of $D_{3} * D_{3}$ with negative genus-increment.

Thus, the genus-increment in this case is -1 .

Research Problem 1. Develop methods for computing the genus distributions when both amalgamated vertices have abitrarily large degrees. For instance, one might augment the present approach with other surgical operations, such as splitting a vertex.

Research Problem 2. Develop methods to solve simultaneous recurrences like (3.3), (3.4) and (4.1), (4.2).

Research Problem 3. As noted in [26], the numbers such as the ones computed in Table 1 and Table 3 appear to support the conjecture that all graphs have unimodal genus distributions. A natural question to ask is whether the vertex-amalgamation of two graphs with unimodal distributions has a unimodal genus distribution.

Research Problem 4. Results of [26] have been successfully used to compute the genus distributions of cubic outerplanar graphs ([10]). Can the results of this paper be similarly used to compute the genus distributions of other non-linear families of graph?

\section{References}

[1] L. W. Beineke, R. J. Wilson, J. L. Gross and T. W. Tucker (editors), Topics in Topological Graph Theory, Cambridge University Press, 2009.

[2] C. P. Bonnington, M. J. Grannell, T. S. Griggs and J. Širáň, Exponential families of nonisomorphic triangulations of complete graphs, J. Combin. Theory (B) 78 (2000), 169-184.

[3] C. P. Bonnington and C. H. C. Little, The Foundations of Topological Graph Theory, Springer, 1995.

[4] J. Chen, J. L. Gross and R. G. Rieper, Overlap matrices and total imbedding distributions, Discrete Math. 128 (1994), 73-94.

[5] Y. C. Chen, Y. P. Liu and T. Wang, The total embedding distributions of cacti and necklaces, Acta Math. Sinica - English Series 22 (2006), 1583-1590.

[6] M. Conder and P. Dobcsányi, Determination of all regular maps of small genus, J. Combin. Theory (B) 81 (2001), 224-242.

[7] M. L. Furst, J. L. Gross and R. Statman, Genus distribution for two classes of graphs, J. Combin. Theory (B) 46 (1989), 22-36. 
[8] L. Goddyn, R. B. Richter, and J. Širán̆, Triangular embeddings of complete graphs from graceful labellings of paths, J. Combin. Theory (B) 97 (2007), 964-970.

[9] M. J. Grannell and T. S. Griggs, A lower bound for the number of triangular embeddings of some complete graphs and complete regular tripartite graphs, J. Combin. Theory (B) 98 (2008), $637-650$.

[10] J. L. Gross, Genus distributions of cubic outerplanar graphs, preprint, 2010, 23 pages.

[11] J. L. Gross, Genus distribution of graphs under surgery: Adding edges and splitting vertices, New York J. of Math. 16 (2010), 161-178.

[12] J. L. Gross and M. L. Furst, Hierarchy for imbedding-distribution invariants of a graph, J. Graph Theory 11 (1987), 205-220.

[13] J. L. Gross, I. F. Khan, and M. I. Poshni, Genus distribution of graph amalgamations: Pasting at root-vertices, Ars Combinatoria 94 (2010), 33-53.

[14] J. L. Gross, D. P. Robbins and T. W. Tucker, Genus distributions for bouquets of circles, $J$. Combin. Theory (B) 47 (1989), 292-306.

[15] J. L. Gross and T. W. Tucker, Topological Graph Theory, Dover, 2001; (original edn. Wiley, 1987).

[16] D. M. Jackson, Counting cycles in permutations by group characters with an application to a topological problem, Trans. Amer. Math. Soc. 299 (1987), 785-801.

[17] D. M. Jackson and T. I. Visentin, A character-theoretic approach to embeddings of rooted maps in an orientable surface of given genus, Trans. Amer. Math. Soc. 322 (1990), 343-363.

[18] D. M. Jackson and T. I. Visentin, An Atlas of the Smaller Maps in Orientable and Nonorientable Surfaces, Chapman \& Hall/CRC Press, 2001.

[19] V. P. Korzhik and H-J Voss, Exponential families of non-isomorphic non-triangular orientable genus embeddings of complete graphs, J. Combin. Theory (B) 86 (2002), 86-211.

[20] J. H. Kwak and J. Lee, Genus polynomials of dipoles, Kyungpook Math. J. 33 (1993), 115-125.

[21] J. H. Kwak and J. Lee, Enumeration of graph embeddings, Discrete Math. 135 (1994), 129151.

[22] J. H. Kwak and S. H. Shim, Total embedding distributions for bouquets of circles, Discrete Math. 248 (2002), 93-108.

[23] L. A. McGeoch, Algorithms for two graph problems: computing maximum-genus imbedding and the two-server problem, $\mathrm{PhD}$ thesis, Carnegie-Mellon University, 1987.

[24] B. Mohar and C. Thomassen, Graphs on Surfaces, Johns Hopkins Press, 2001.

[25] B. P. Mull, Enumerating the orientable 2-cell imbeddings of complete bipartite graphs, J. Graph Theory 30 (1999), 77-90.

[26] M. I. Poshni, I. F. Khan and J. L. Gross, Genus distribution of graphs under edgeamalgamations, Ars Math. Contemp. 3 (2010) 69-86.

[27] S. Stahl, Region distributions of graph embeddings and Stirling numbers, Discrete Math. 82 (1990), 57-78.

[28] S. Stahl, Permutation-partition pairs III: Embedding distributions of linear families of graphs, J. Combin. Theory (B) 52 (1991), 191-218.

[29] S. Stahl, Region distributions of some small diameter graphs, Discrete Math. 89 (1991), 281 299.

[30] E. H. Tesar, Genus distribution of Ringel ladders, Discrete Math. 216 (2000) 235-252. 
[31] T. I. Visentin and S. W. Wieler, On the genus distribution of $(p, q, n)$-dipoles, Electronic J. of Combin. 14 (2007), Art. No. R12.

[32] L. X. Wan and Y. P. Liu, Orientable embedding distributions by genus for certain types of graphs, Ars Combin. 79 (2006), 97-105.

[33] L. X. Wan and Y. P. Liu, Orientable embedding genus distribution for certain types of graphs, J. Combin. Theory (B) 47 (2008), 19-32.

[34] A. T. White, Graphs of Groups on Surfaces, North-Holland, 2001. 